\title{
Incidencia de Listeria spp. en carcasas de pollo congelado en un supermercado del nororiente de Bogotá
}

\author{
Claudia Pérez-Rubiano', Marcela Mercado-Reyes², \\ Ana Karina Carrascal-Camacho'

\begin{abstract}
1. Laboratorio de Microbiología de Alimentos. Grupo de Biotecnología Ambiental e Industrial. Departamento de Microbiología, Facultad de Ciencias, Pontificia Universidad Javeriana, Bogotá Colombia.

2. Grupo de enfermedades infecciosas, Pontificia Universidad Javeriana, Bogotá Colombia.

Correspondencia: acarrasc@javeriana.edu.co

Recibido: 08-08-08 / Aceptado 15-10-08
\end{abstract}

\section{Resumen}

Listeria spp. es un género bacteriano que contamina alimentos de origen animal, incluido el pollo, yl puede permanecer viable durante las cadenas de producción y distribución. En el presente trabajo se analizaron 9l muestras de carcasas de pollo, obtenidas en una distribuidora en el nororiente de Bogotá; las muestras se tomaron en un período de 9 semanas y luego fueron procesadas. De las 91 carcasas, 40 (43.95\%) resultaron positivas para Listeria spp. La presencia de este microorganismo puede estar asociada a deficiencias en los sistemas de tecnología de limpieza en las plantas de beneficio y/o contaminación con utensilios durante el desprese.

Palabras clave: biopelícula, carcasas de pollo, industria cárnica, Listeria spp., microorganismos psicrótrofos, Pseudomonas.

\section{Abstract \\ Incidence of Listeria spp., in frozen poultry carcasses at supermarket in northwest Bogotá}

Listeria spp. it is a bacterial gene that contaminates foods of animal origin including poultry, where it can remain viable during the chains of production and distribution. In this work, 91 samples of chicken carcasses were analyzed; they were obtained in a distributor in northwest Bogota. The samples were taken in a period of 9 weeks and processed shortly thereafter. From the 9I carcasses, 40 (43.95\%) were positive for Listeria spp. The presence of this microorganism can be associated to deficiencies in the systems of cleaning technology in the poultry factory and/or contamination with utensils during cut-up.

Key words: biofilm, Listeria spp., meat industry, poultry carcasses, Pseudomonas, psicrotrofo. 


\section{Introducción}

El género Listeria spp. se encuentra ampliamente distribuido en la naturaleza, incluyendo como reservorios una gran cantidad de mamíferos, aves, crustáceos y peces $(1,2)$. También se ha encontrado en las plantas de beneficio y procesamiento de alimentos, donde se ha convertido en un problema complejo por su persistencia en superficies, pisos y sifones $(2,3)$. Este género bacteriano puede crecer en un amplio rango de temperatura y permanecer metabólicamente activo a $-1,5^{\circ} \mathrm{C}(4,5)$. Dentro de este género bacteriano se ha reconocido la importancia que tiene Listeria monocytogenes, ésta puede ser transmitida a los humanos a través del consumo de alimentos contaminados. Durante los últimos años este microorganismo ha sido objeto de diversos estudios, en especial en la industria cárnica, ya que su alta tasa de mortalidad (20-30\%), lo convierte en un riesgo potencial para los consumidores $(4,6)$.

Listeria spp. posee la capacidad de sobrevivir durante períodos prolongados en el ambiente (suelo, plantas y agua) y multiplicarse a temperatura de refrigeración. En general, este género puede sobrevivir en el interior o en la superficie de los alimentos durante largos períodos en condiciones adversas, presentan la habilidad de adherirse a las superficies formando biopelículas, por lo que pueden generar resistencia a los procesos de limpieza y a los desinfectantes. Esta condición ha hecho que la presencia de este género se considere como un indicador de prácticas de saneamiento inadecuadas para la industria de alimentos en la última década (7).

L. monocytogenes es una bacteria patógena humana que ha sido asociada con productos crudos de pollo (posible vector en la transmisión de la listeriosis) y en la última década con el grupo denominado "ready to eat" (listos para consumo) $(4,6)$. Mientras su morbilidad es baja, este microorganismo puede causar alta mortalidad al ser comparado con otras enfermedades transmitidas por el consumo de pollo contaminado con Salmonella o Campylobacter (8). Listeria puede estar presente dentro de la planta procesadora llegando a contaminar en algún momento las superficies de contacto con los alimentos, y al producto mismo. Este género bacteriano logra formar nichos ecológicos con otras bacterias como Pseudomonas, permitiendo su persistencia en el medio ambiente y haciendo difícil su erradicación una vez se ha establecido $(9,10)$. De esta manera puede llegar a contaminar los pollos durante el proceso de beneficio y desposte (11).

En la industria avícola se han encontrado como puntos de contaminación pisos, drenajes, mangueras, equipos de acero inoxidable entre otros (12), por lo que deben mantenerse normas estrictas durante el proceso de beneficio y desprese, para reducir el riesgo de contaminación. Por lo expuesto anteriormente, el objetivo de este trabajo fue determinar la incidencia de Listeria spp., en carcasas de pollo en una distribuidora del nororiente de Bogotá.

\section{Materiales y métodos}

Las muestras se tomaron del punto de venta de una distribuidora de pollos del nororiente de Bogotá. El número de muestras de pollo se determinó de acuerdo al programa estadístico tamaño de muestra 1.0, con una prevalencia esperada de 33\%, una diferencia máxima esperada del $10 \%$ y un error tipo I del 5\%. El total de muestras analizadas fueron 91, que se tomaron en un periodo de 9 semanas, con un promedio de 10 muestras por semana. Se escogieron los pollos congelados al azar, éstos se llevaron refrigerados y se permitió su descongelación en condiciones de refrigeración antes de su procesamiento con el fin de reducir el impacto por injuria bacteriana. Durante la toma de las muestras se midió la temperatura interna de los pollos con el fin de establecer las condiciones de almacenamiento (13).

\section{Análisis microbiológico}

Las carcasas de pollo se analizaron tomando como referencia la técnica de Lee y MacClain ó método de la USDA-FSIS basado en la norma ISO 11290-1 con algunas modificaciones en los medios de cultivo y las condiciones de enriquecimiento de las muestras $(14,15)$. La carcasa de pollo se removió asépticamente y se colocó en bolsas de plástico estériles, luego se adicionaron $300 \mathrm{~mL}$ de agua peptonada tamponada a la bolsa y se agitó unas cincuenta veces (14). De esta suspensión se tomaron 25 $\mathrm{mL}$ y se adicionaron $225 \mathrm{~mL}$ de caldo de enriquecimiento Listeria - LEB (Oxoid, UK). Las muestras se incubaron por 4 horas a $30^{\circ} \mathrm{C}$, se adicionaron suplementos selectivos (SR 140E, Oxoid) y luego se incubaron por 20h (14). Posteriormente $0.1 \mathrm{~mL}$ de LEB se transfirió a $10 \mathrm{~mL}$ de 
caldo de enriquecimiento Listeria - LEB (Oxoid, UK) y se incubó a $30^{\circ} \mathrm{C}$ por 24 a 30 horas.

Una asada del caldo se estrió en agar Oxford y agar Palcam, se incubó a $35^{\circ} \mathrm{C}$ por $48 \mathrm{~h}(13,14)$. Transcurrido el tiempo de incubación se seleccionaron tres colonias que presentaron hidrólisis de la esculina, se aislaron sobre agar TSAYE y se incubaron a $35^{\circ} \mathrm{C}$ por 24 horas (16). Una vez purificada la cepa se sometió a identificación bioquímica, incluyendo: la prueba de iluminación de Henry, catalasa y coloración de Gram (17). Se consideró como positiva al género Listeria las muestras que presentaron iluminación de Henry positiva, catalasa positiva y bacilos Gram positivos. La incidencia se estimó usando la siguiente fórmula (18).

$$
\text { Incidencia }=\frac{\# \text { Listeria }_{\text {muestras }}}{\text { Total }_{\text {muestras }}} \times 100
$$

\section{Resultados}

De las 91 muestras procesadas utilizando el método tradicional, se obtuvieron 40 muestras correspondientes al género Listeria spp., correspondiendo a una incidencia del $43.95 \%$. La temperatura promedio de los pollos congelados estuvo en $-15^{\circ} \mathrm{C}$. Durante la investigación se encontró una gran interferencia en la recuperación de este microorganismo asociada a la presencia de Pseudomonas (datos no mostrados), por lo que fue necesario adaptar la metodología, adicionando una mayor cantidad de los agentes inhibitorios propios del medio, Figura1.

\section{Discusión}

La incidencia de Listeria spp., obtenida en este estudio fue del $43.95 \%$; estos resultados demuestran que el microorganismo se encuentra contaminando pollos congelados que están listos para su comercialización. Estos resultados difieren con otros estudios realizados, Karoley y col. (2005) encontraron una incidencia del 14,89\% de Listeria spp en pollo, donde el 8,5\% correspondió a $L$. monocytogenes (11). Nierop y col. (2005) encontraron un porcentaje de Listeria de 19.2\% en carcasas crudas de pollo (19); Pelliser y col. (2001), encontraron un $29 \%$ en carcasas de pollo crudo (15); contrastando con los datos obtenidos por Galli y colaboradores, en carcasas de pollo (36\%) (20), lo cual podría explicarse como parte de la ecología propia del microorganismo y su amplia distribución a nivel de instalaciones (7), o estar relacionado con el número de muestras analizadas y la técnica de aislamiento que pueden reducir o aumentar el número de cepas recuperadas, generando incertidumbre en los datos obtenidos.

En Colombia existen datos de incidencia en carne de pollo refrigerado que son más bajos a los obtenidos en esta investigación. Bernal reportó una incidencia de L. monocytogenes del 33,33\% en un estudio realizado en Santiago de Cali (13), otro estudio llevado a cabo en el 2007 por Carrascal y colaboradores mostraron incidencias de 14,5\% de Listeria spp. en pollo beneficiado en 255 muestras en un intervalo de tiempo similar a esta investigación (21), por lo que se puede inferir que el número de muestras analizadas afecta los resultados obtenidos, ya que un mayor número de muestras puede siempre generar datos más confiables. Si bien se esperaba encontrar un número menor de Listeria, debido a la temperatura de congelación utilizada, se ha logrado establecer que ésta puede mantenerse viable en carnes de pollo congeladas (22), y que la contaminación en estas condiciones puede ser incluso mayor que en pollo refrigerado, debido a que estos son más propensos a la contaminación durante el procesamiento y almacenamiento (22).

Como se mencionó anteriormente la naturaleza ubicua de este género le permite contaminar las plantas

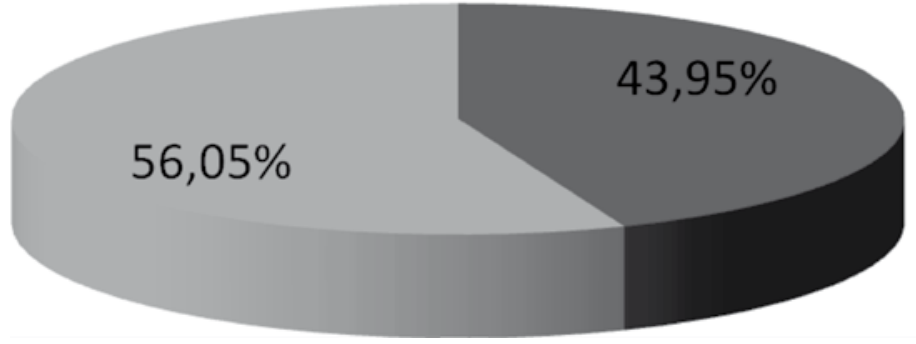

Presencia de Listeria spp.

Figura 1. Incidencia de Listeria spp. en carcasas de pollo.

Ausencia de Listeria spp. 
en donde las condiciones higiénicas no son las adecuadas $(6,23)$, logrando colonizar salas de proceso como: el preenfriamiento (Prechiller), enfriamiento (chiller) y desprese, debido a que estas operaciones se llevan a cabo en tanques y con cuchillas elaborados en acero inoxidable, donde Listeria spp., puede encontrarse. La capacidad que tiene de formar fácilmente biopelículas, se convierte en focos potenciales de diseminación dentro de la planta, siendo muy difícil su eliminación, de ahí la importancia de tener establecido programas de limpieza y desinfección eficaces contra este género microbiano, ya que su presencia será indicador de fallas en este proceso $(23,24)$.

El agua y el hielo empleado en los tanques son factores de riesgo que pueden generar la proliferación de Listeria spp., por eso el agua que se emplea debe ser potable, se debe cambiar continuamente y mantener un constante monitoreo de su temperatura para evitar la contaminación por este microorganismo (25). La "Federal Drug Administration" (FDA) ha establecido el uso de soluciones de cloro entre $50 \mathrm{ppm}$ y $150 \mathrm{ppm}$, para la reducción del microorganismo en el agua de chiller con un tiempo máximo de residencia de 1 hora (26). En Colombia la resolución 4287 del Ministerio de Protección Social, no especifica cantidades de desinfectantes, aunque hace énfasis en la importancia de su uso (25).

Con respecto a la técnica empleada, fue necesario reducir el tiempo de incubación del enriquecimiento primario en 24 horas, ya que se observó el crecimiento masivo de Pseudomonas spp., en las cajas con agar Palcam y Oxford a pesar de haber utilizado antibióticos en los medios de cultivo. Esto demuestra una vez más que las técnicas desarrolladas en otros países no siempre pueden aplicarse de la misma manera en nuestro entorno, ya que la ecología de los alimentos difiere significativamente. Estos resultados concuerdan con los datos de Gallegos y colaboradores en el 2007, quienes tuvieron que aumentar las concentraciones de antibióticos para poder recuperar cepas de Listeria spp. (27). De igual manera Carrascal y colaboradores, en su investigación tuvieron problemas en las semanas iniciales del muestreo, ya que la presencia de Pseudomonas impidió la recuperación de Listeria en el laboratorio (21).

De otro lado, se ha logrado establecer que Pseudomonas es un gran competidor de microorganismos como Listeria y que puede inhibir su recuperación en los medios de cultivo aunque esté presente en los alimentos $(22,28)$. Estos datos demuestran una alta circulación de este microorganismo en las carcasas de pollo. La presencia de Pseudomonas en la industria avícola se ha utilizado como indicador de la alteración del pollo en bandeja refrigerada, ya que puede causar malos olores cuando la concentración alcanza $10^{7} / \mathrm{cm}^{2}(28)$. Este microorganismo puede estar dentro de la plantas de alimentos debido a su alta resistencia a desinfectantes y a su habilidad para formar biopelículas $(9,29)$. Además, su crecimiento se ve favorecido por la alta humedad y la bajas temperaturas (9), condiciones que se dieron en esta investigación.

La presencia de Pseudomonas en las carcasas de pollo se pueden asociar a deficiencias en los programas de limpieza y desinfección, donde el factor relevante es el uso de concentraciones inadecuadas del desinfectante, permitiendo generar cepas resistentes que crean nichos ecológicos dentro de las diferentes zonas de la planta de sacrificio (30). Muchas cepas son psicrofílicas por lo que pueden mantenerse aún a bajas temperaturas (9). El punto crítico donde la carne de pollo se contamina con Pseudomonas y Listeria es durante el desplumado, allí los microorganismos alterantes o patógenos, se ubican en la superficie y en la piel. Las superficies de las aves de corral frescas conservadas en un ambiente de humedad elevada son sensibles al crecimiento de bacterias aeróbicas como Pseudomonas. Estos organismos crecen bien en las superficies, donde forman colonias diminutas que después confluyen para producir la viscosidad característica de las canales de aves de corral alteradas (31).

Los microorganismos psicrotróficos que se encuentran en carcasas de pollo son heterogéneos, encontrándose géneros como: Flavobacterium spp., Shewanella putrefaciens, Acinetobacterspp., Corynebacteriium spp., Moraxella spp. y Pseudomonas spp. $(31,32)$. Las especies de Pseudomonas compiten con éxito en los alimentos musculares, almacenados aeróbicamente en refrigeración por varias razones: poseen una velocidad de crecimiento competitiva, incluso a temperaturas de refrigeración, crecen en todo el intervalo de $\mathrm{pH}$ de los alimentos musculares (5.5 a 7.0), mientras que muchas bacterias, por ejemplo, Moraxella spp. y Acinetobacter spp., no son competidoras bajo temperaturas de refrigeración y a valores de $\mathrm{pH}$ menores 
del intervalo citado (32-34). Esto también se explicaría para la presencia de Listeria, por su habilidad para crecer a bajas temperaturas $(4,6,31)$. Este aspécto es el parámetro ambiental más importante que influye en el crecimiento de los microorganismos de los alimentos musculares. Cuando la temperatura está por debajo de la óptima de crecimiento de los microorganismos, aumenta la duración de los tiempos de generación y latencia y, en consecuencia, se identifica el crecimiento. A medida que disminuye la temperatura hasta la mínima de crecimiento, continúa alargándose el tiempo de latencia hasta que finalmente cesa la multiplicación (7). Los estudios acerca de la carga bacteriana en las canales frescas de aves de corral revelan que en ellas se pueden encontrar más de 25 géneros microbianos. No obstante cuando estas carnes se alteran a bajas temperaturas, la mayoría de los autores coinciden en que los principales microorganismos causantes de la misma pertenecen al género Pseudomonas spp. (33).

Las superficies de las aves recién sacrificadas y conservadas en un ambiente de humedad, son sensibles al crecimiento de bacterias aeróbicas como Pseudomonas, las cuales compiten por espacio y por nutrientes con Listeria spp. y otros microorganismos psicrótrofos, de ahí que es posible que los datos de Listeria de este estudio sean incluso superiores, a los obtenidos en otras investigaciones (21). Por ultimo, un factor que pudo contribuir a la presencia de Listeria spp. en el pollo congelado, son las inadecuadas condiciones higiénicas en la cadena agroalimentaria, incluyendo su almacenamiento, transporte, beneficio, distribución y comercialización, que no necesariamente reflejan las condiciones generales del país $(21,24)$. Es importante señalar que una incidencia tan alta en este tipo de productos, pone de manifiesto la ausencia de un buen programa de limpieza y desinfección durante el proceso, por lo que será necesario reforzar, a nivel de plantas de beneficio, los programas dirigidos a la reducción de este género bacteriano, particularmente de la L. monocytogenes, que por su alta tasa de mortalidad es de importancia en salud pública (4). $\mathbf{N} \mathbf{V}$

\section{Referencias}

1. Hof H. History and epidemiology of listeriosis. FEMS. 2003;35:199-202.

2. Torres KJ, Sierra SC, Poutou RA, Vera H, Carrascal AK, Mercado M. Incidencia y diagnóstico de Listeria monocytogenes; microorganismo zoonótico emergente en la industria de alimentos. Rev UDCA actualidad y divulgación científica. 2004;7:25-57.

3. Hong Y-H, Ku G-J, Kim M-K, Bing K. Inactivation of Listeria monocytogenes and Campylobacter jejuni in chicken by aqueous chlorine dioxide treatment. J Food Sc Nutr. 2007;12:279-283.

4. Food Safety Authority of Ireland (FSAI). The control of management of Listeria monocytogenes contamination of food Dublin. 2005.

5. Kyung J, D'sa E, Harrison MA, Harrison JA. Listeria monocytogenes survival in refrigerator dill pickles. J Food Prot. 2005;68:2356-2361.

6. FAO/OMS. World Health Organization/Food and Agriculture Organization of the United Nation. Risk assessment of Listeria monocytogenes in ready-to-eat foods. Technical report. Microbiological risk assessment series 5. Rome, Italy. 2004.

7. Loura C, Almeida R, Almedia P. The incidence and level of Listeria spp and Listeria monocytogenes contamination in processed poultry and at poultry processing plant. J Food Saf. 2005;25:19-25

8. Gutiérrez A, Paasch L, Calderón N. Salmonelosis y campylobacteriosis las zoonosis de mayor expansión en el mundo. Vet Mex. 2008;39:81-90.

9. Carpenter B, Chassaing D. Interactions in biofilms between Listeria monocytogenes and resident microorganisms from food industry premises. Int J Food Microbiol. 2004;97:111-122.

10. Román M, Tirado S. Evaluación de la formación de biopelículas de cepas de Listeria monocytogenes y Pseudomonas spp. en empaques utilizados en la industria de alimentos. In: Departamento de Microbiología. Carrera de Microbiología Industrial. Pontificia Universidad Javeriana: Bogotá, D.C. 2004

11. Karoley D, Barbuddhe N, Kurjure V, Gunjal P. Prevalence of Listeria monocytogenes in poultry meat in Vidharga region of India. In: XVII ${ }^{\text {th }}$ European Symposium on the Quality of Poultry Meat. Doorwerth. The Netherlands; 2005.

12. Lawrence LM, Gilmour A. Incidence of Listeria spp. and Listeria monocytogenes in a poultry processing environment and in poultry products and their rapid confirmation by multiplex PCR. Appl Environ Microbiol. 1995;61:847.

13. Bernal L. Incidencia de Listeria monocytogenes en carne de res, cerdo y pollo en Santiago de Cali. In: Departamento de microbiología. Carrera de Bacteriología. Pontificia Universidad Javeriana Bogotá, D.C.1997.

14. McClain D, Lee WH. Development of USDA-FSIS method for isolation of Listeria monocytogenes from raw meat and poultry. $\mathrm{J}$ Assoc Off Anal Chem. 1988;71:660-644.

15. Pelisser M, Mendez S, Sutherland A. Detection of Listeria species in refrigerated chicken carcasses using cleaview and a modified conventional culture method. Braz J Microbiol. 2001;32:113-116.

16. Burbano EM, Carrascal AK, Mercado M, Poutou RA. Validación de PCR para Listeria monocytogenes en leches. Normas y Calidad. 2003;57:39-48. 
17. United States Department of Agriculture (USDA). Microbiology Laboratory Guidebook: food safety and inspection service, officinal of public health and science: isolation and identification of Listeria monocytogenes from read, meat, poultry, egg, and environmental. pg 22. 2002.

18. Greemberg R, Daniela S, Flanders D, Eley J Boring J. Epidemiología clínica. México D. F. Ed Manual Moderno, 4 ed, 2005.

19. Nierop W, Duse A, Marais E, Aithma N, Thothobolo N, Kassel M, Stewart R, Potgieter A, Fernandes B, Galpin J, Bloomfield S. Contamination of chicken carcasses in Gauteng, South Africa, by Salmonella, Listeria monocytogenes and Campylobacter. Int J Food Microb.2005;99:1-6.

20. Galli R, Sannipoli CGT, Valente C. Listeria monocytogenes quale contaminate di carcasse di pollo. Ind. Aliment. 1992;31:21-24.

21. Carrascal AK, Correa C, Fonseca J. Detección de Listeria monocytogenes en expendios de pollo procesado en la zona suroccidental de Bogotá, D.C. Lab Actual. 2007;23:29-32.

22. Mahmood MS, Ahmed AN, Hussain I. Prevalence of Listeria monocytogenes in poultry meat, poultry meat products and other related inanimated at Faisalabad. Pakistan J Nut. 2003;2:346-349.

23. Frank JF, Koffi RA. Surface Adherent Growth of Listeria monocytogenes is Associated with Increased Resistance to Surfactant Sanitizers and Heat. J Food Prot. 1995;53:550-554.

24. Lunden J, Autio TR, Korkeala HL. Transfer of persistent Listeria monocytogenes contamination between food processing plants associated with a dicing machine. J Food Prot. 2002 65:1129-1133.

25. Ministerio de Protección Social. Resolución 4287 pg. 47; 2007.
26. US Federal Register.. 21 CFR part 173. Secondary direct food additives permitted in food for human consumption. pg117; 1996.

27. Gallegos J, Arrieta G, Mattar S, Poutou R, Trespalacios A, Carrascal AK. Frecuencia de Listeria spp en quesos colombianos costeños. Rev MVZ Córdoba. 2007;12:996-1012.

28. Buchanan RL, Bagi LK. Microbial competition: effect of Pseudomonas fluorescens on the growth of Listeria monocytogenes. Food Microbiol. 1999;16:523-529.

29. Fung D. Métodos rápidos y automatización en microbiología alimentaria. II Workshop. Facultad de veterinaria. Universidad Autónoma de Barcelona. 2003.

30. Sander J, Hofacre J, Cheng I, Wyatt R. Investigation of resistance of bacterial from commercial poultry sources to commercial disinfectants. Avian Diseases. 2002;46:997-1000.

31. Langsrud S, Sundheim S. Factors contributing to the survival of poultry associated Pseudomonas spp. Exposed to a quaternary ammonium compound. J Appl Microbiol. 1997;82:705-712.

32. Sundheim A, Sletten A, Dainty RH. Identification of Pseudomonads from fresh and chill-stored chicken carcasses. Int J Food Microbiol. 1998;39:185-194.

33. Arnaut-Rollier I, De Zutter L, Van Hoof J. Identities of the Pseudomonas spp. in flora from chilled chicken. Int J Food Microbiol. 1999;48:87-96.

34. Hinton A Jr, Cason JA, Ingram KD. Tracking spoilage bacteria in commercial poultry processing and refrigerated storage of poultry carcasses. Int J Food Microbiol. 2004;91:155-165. 